\title{
Evaluation of Minimum Variance Distortionless Response Beamforming Algorithm Based Circular Antenna Arrays
}

\author{
Suhail Najm Shahab ${ }^{1}$, Ayib Rosdi Zainun ${ }^{1}$, Balasim S. S. ${ }^{2}$, Nurul Hazlina Noordin ${ }^{1}$ \& Izzeldin Ibrahim \\ Mohamed $^{1}$ \\ ${ }^{1}$ Faculty of Electrical and Electronics Engineering, University Malaysia Pahang, Pahang, Malaysia \\ ${ }^{2}$ Electrical Department, College of Engineering, University of Anbar, Iraq \\ Correspondence: Suhail Najm Shahab, Faculty of Electrical and Electronics Engineering, University Malaysia \\ Pahang, 26600, Pahang, Malaysia. Tel: 60-146-279-045. E-mail: 68suhel@gmail.com
}

Received: October 16, 2015

Accepted: November 1, 2015

Online Published: September 16, 2016

doi:10.5539/mas.v11n1p1

URL: http://dx.doi.org/10.5539/mas.v11n1p1

\begin{abstract}
Wireless data traffic is in a continuous growth, and there are increasing demands for wireless systems that provide deep interference suppression and noise mitigation. In this paper, adaptive beamforming (ABF) technique for Smart Antenna System (SAS) based on Minimum Variance Distortionless Response (MVDR) algorithm connected to Circular Antenna Array (CAA) is discussed and analyzed. The MVDR performance is evaluated by varying various parameters; namely the number of antenna elements, space separation between the elements, the number of interference sources, noise power label, and a number of snapshots. LTE networks allocate a spectrum band of $2.6 \mathrm{GHz}$ is used for evaluating the MVDR performance. The MVDR performance is evaluated with two important metrics; beampattern and SINR. Simulation results demonstrate that as the antenna elements increase, the performance of the MVDR improves dramatically. This means the performance of MVDR greatly relies upon the number of the elements. Half of the wavelength is considered the best interelement spacing, the performance degraded as noise power increased, and more accurately resolution occurred when the number of snapshots increased. The proposed method was found to be performed better than some existing techniques. According to the result, the beampattern relies on the number of element and the separation between array elements. Also, the SINR strongly depends on noise power label and the number of snapshots.
\end{abstract}

Keywords: adaptive antenna array, beamforming, circular antenna array, LTE, minimum variance distortionless response, MVDR, smart antenna

\section{Introduction}

Currently, the mobile cellular networks are experiencing a massive evolution of data traffic, because of multimedia and internet applications that are used by a vast number of devices such as smartphones, mobile PC and tablets (Cisco Visual Networking Index, 2014; Ericsson Mobility Report, 2015). Most of the beamforming (BF) algorithms have been considered to be used at the base station (BS) side, because the array of antennas are not applicable at mobile terminals due to space limitations (Liberti \& Rappaport, 1999).

A smart antenna system (SAS) is an adaptive antenna array (AAA) with smart digital signal processing algorithms used to identify spatial signal signature such as direction of arrival (DoA) of the signal, and used it to calculate BF vectors, to track and locate the antenna beams on the desired target (Jacobsen, 2001; Web ProForum Tutorials, 2006; Winters, 2000). SASs are customarily categorized as either switched beam or adaptive array systems. The AAA system consists of a number of the array elements which should be relatively low, in order to avoid unnecessarily high complexity in the signal processing unit. The array configuration can scan either one or two dimensions, depending on the dimension of space to be accessed. In practice, the array geometry can take either one of the following realizations; linear antenna array (LAA) or planar (PAA) or circular (CAA) or rectangular (RAA) or cubic (CuAA) (Stevanovic, Skrivervik \& Mosig, 2003).

The motivation behind using an adaptive approach is also due to the need of adaptive beamforming (ABF) in wireless communication applications (S. Das, 2008; El-Keyi \& Champagne, 2008; Hong, Huang, Chiu \& Kuo, 2007; Sun, Hirata, Ohira \& Karmakar, 2004). Examples of AAAs (R. Saunders \& A. Zavala, 2007) are widely used in microphone array, radar, sonar, medical imaging, seismology, radio astronomy, medical imaging, speech 
processing, and wireless communications. An AAA is designed to optimize the beampattern according to specific criteria such as minimum variance, maximum entropy, maximum SINR (Godara, 2004). For instance, the Capon (Capon, 1969) beamformer minimizes array output power subject to a linear constraint, known as the look direction constraint, which ensures a desired response from a specific direction. With the increasing trend of the number of subscribers and demand for different services in wireless systems, there are always requirements for better coverage, higher data rate, reduced operating cost and improve spectrum efficiency. To achieve these requirements beyond the Long Term Evolution (LTE) which is introduced by the 3rd Generation Partnership Project (3GPP), beamforming technique is able to focus the array antenna pattern into a particular direction and thereby enhances the signal strength.

Interference is one of the significant obstacles in the cellular systems. The interference can be caused by other users or by the signal itself due to multipath components (Halim, 2001). The signal is gathered with another version of the signal that is delayed because of another propagation path. The fundamental principle of ABF is to track the statistics of the surrounding interference and noise field as well as adaptively seek for the best position of the nulls that reduce the unwanted signal under the constraint of not distorted the desired signal at the beamformer's output (Pan, Chen \& Benesty, 2014).

One of the popular method for ABF is introduced by Capon (Capon, 1969) known as Minimum Variance Distortionless Response (MVDR). The fundamental purpose of the MVDR is to estimates the beamforming coefficients in an adaptive way by minimizing the variance of the residual noise and interference while enforcing a set of linear constraints to ensure that the real user signals are not distorted (Pan et al., 2014). The authors in (Khaldoon, Rahman, Ahmad \& Hassnawi, 2014) proposed an enhanced model of MVDR algorithm by changing the position of the reference element in steering vector to be in the middle of the array with an odd number of elements. Their results show that modified MVDR has a realistic behavior, especially for detecting the incoming signals direction and outperforms the conventional MVDR. The signal to interference plus noise ratio (SINR) maximization is one of the criterion employed in joint transmitter and receiver BF algorithms (Choi, Murch \& Letaief, 2003; Kum, Kang \& Choi, 2014; Serbetli \& Yener, 2004). In (Manolakis, Ingle \& Kogon, 2005) mentioned that the spacing between adjacent element $(d)$ must be greater than or equal to the half of the signal wavelength $(\lambda)$ to avoid spatial aliasing. In (K. J. Das \& Sarma, 2012), the author presents a comparative study of minimum variation distortionless algorithm and LMS algorithm. Where results show that LMS is the better performer. In an analysis of (Rao \& Sarma, 2014) discussed the mixing of different algorithm based linear antenna array is applied to control the level of side lobes and null in the unwanted direction. They found the maximum null depth of $-63 \mathrm{~dB}$ by using 20 elements. The researchers in (Pan et al., 2014) investigate the performance of the MVDR beamformer for four different type of noise and source incidence angles using SNR and beampattern as the evaluation criteria. An evaluation of the tradeoff between reverberation and reduce the noise of the MVDR is presented by (Habets, Benesty, Cohen, Gannot \& Dmochowski, 2010). Research effort proposed by (Han, 2009) to optimize the output pattern of the antenna array system using genetic algorithm. The optimization creation is based on output beampatterns with low side lobes by finding the best amplitude layout of antenna elements that produce low side lobe levels.

SAS includes signal processing capabilities that perform tasks like the DOA estimation of the incoming signals and then SAS can adjust the antenna itself using beamforming techniques to achieve better transmission or reception beampattern which increase SINR by mitigating co-channel interference present in the wireless communication system (El Zooghby, 2005). A SAS that is held in the BS of a mobile system comprises of an array of antennas where the amplitudes are accustomed to a group of weight vectors using an ABF algorithm (Gross, 2015). The ABF algorithm improves the output of the array beampattern in a way which it maximizes the radiated power where it will be produced in the direction of the real user. Moreover, deep null is produced in the interfernce directions that mitigate co-channel interference from other users in the neighboring cells. Before ABF, the direction of arrival estimation is used to specify the main directions of users and interferers. The function of $\mathrm{ABF}$ algorithms is used to direct the main beam with unity gain towards the Signal of Interest (SOI) direction whilst negative power (null) in the direction of Signal not of Interest (SNOI) (Balanis \& Ioannides, 2007; Godara, 2004). Recently, the antenna arrays have been widely used to enhance the performances for the wireless communication system, where the antenna array allows to improve the coverage area in cellular communication as well the satellite system (Khraisat, 2012).

So far, $\mathrm{ABF}$ is a function of the number antenna elements, separation between adjacent elements, angular separation between desired user direction and undesired signal directions, noise power level as well as a number of snapshots. Therefore, it is important to investigate the impact of these parameters on the radiation beampattern of an antenna array that able to offer the best BF capabilities in terms of directing the main beam toward the 
direction of the SOI while placing nulls towards the direction of SNOI. However, most existing literatures investigate the MVDR beamformer without focusing on all parameters that affecting the nulling level and overall MVDR performance. This paper focus on the impact of these parameters such as the number of antenna elements, space separation between the elements, the number of interference source, noise power label, and a number of snapshots based on CAA geometry (Belloni \& Koivunen, 2006; Mathews \& Zoltowski, 1994; Van Trees, 2002). The analysis of the MVDR in this work is carried out in four different scenarios where the MVDR performance is evaluated with two important metrics; beampattern and SINR. This analysis not only helps to understand the MVDR beamformer, but also helps to design better array systems in actual application. The rest of this paper is organized as follows. In section 2, MVDR beamformer based on circular antenna array design method with signal propagation model is described. The simulation results and performance analysis are provided in Section 3 , this section is divided into four sub-sections based on the comparison parameters is taken. Finally, in Section 4, the paper's conclusions and summary of MVDR performance are described.

\section{System Model \& MVDR Beamforming}

In this section, the mathematical formulation of the design model for ABF will be presented in detail. Consider a single cell with $L$ elements antenna array. Let there be $S$ desired user signal and $I$ interference sources transmitting on the same frequency channel simultaneously. The algorithm begins by creating a real-life signal model. Consider a number of plane waves from $K$ narrowband sources impinging from different angles $(\theta, \phi)$, the impinging radio frequency signal reaches into the antenna array from a far field to the array geometry in a CAA and all elements are distributed around a circle of radius, $r$. If the interelement distance is constant, it is called Uniform (UCAA). Figure 1 show a typical CAA geometry added to BF system. However, each signal multiplied by adaptable complex weight vector and then summed to form the output beampattern.

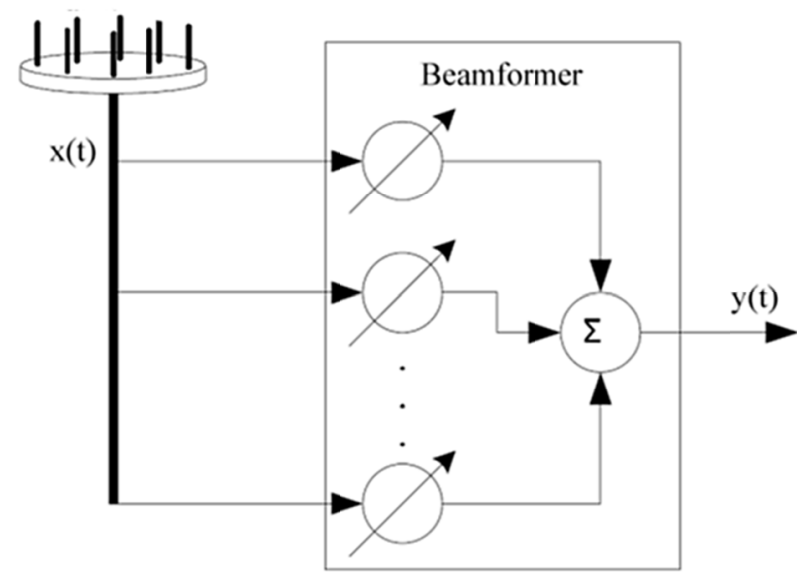

Figure 1. Circular antenna array geometry combines with beamforming system

The total signals received by the AAA at time index, $t$, become:

$$
x_{T}(t)=\sum_{s=1}^{S} x_{s}(t) a\left(\theta_{s}\right)+\sum_{i=1}^{I} x_{i}(t) a\left(\theta_{i}\right)+x_{n}
$$

Where $x_{T}(t) \in C^{K \times L}, x_{s}(t), x_{i}(t), x_{n}(t)$, denote the desired signal, interference signal and noise signal added from White Gaussian noise, respectively. The unwanted signal consists of $x_{i}(t)+x_{n}(t)$ and $I$ is the number of interferen-ce, the desired angle and interference direction of arrival angles are $\left(\theta_{s}, \phi_{s}\right)$ and $\left(\theta_{i}, \phi_{i}\right), i=1,2 \ldots, I$, respectively. $a\left(\theta_{s}, \phi_{s}\right)$ denote the steering vector for wanted signal while $a\left(\theta_{i}, \phi_{i}\right)$ refers to the interference signal steering vector.

Steering vector is a complex vector $\in \boldsymbol{C}^{L \times K}$ containing responses of all elements of the array to a narrowband source of unit power depending on the incident angle, which is given by (Belloni \& Koivunen, 2006; Mathews \& Zoltowski, 1994):

$$
a(\theta, \phi)=\left[e^{-j q r \sin (\theta) \cos (\phi-2 \pi l / L)}\right]
$$




$$
A=\left[a_{0}, a_{1}, a_{2}, \ldots a_{L-1}\right]^{T}
$$

Where $j$ is the imaginary unit of a complex number, (i.e. $j^{2}=-1$ ), $q=2 \pi / \lambda$ is the wave number, $r=L d / 2 \pi$ is the radius of the circular antenna array, $\lambda$ is the carrier wavelength, $(\theta, \phi)$ composed of azimuth angle $\in[0,2 \pi]$ and elevation angle $\in[0, \pi / 2]$ while $A$ represent the matrix form of the steering vector and (. $)^{T}$ denote the transposes operators.

The signal $x_{T}(t)$ received by multiple antenna elements is multiplied by a series of amplitude and phase (weight vector coefficients) which accordingly adjust the amplitude and phase of the incoming signal. This weighted signal is a linear combination of the data at $L$ elements, resulting in the array output, $y(t)$ at any time $t$, of a narrowband beamformer, which is defined as:

$$
y(t)=\sum_{l=1}^{L} w^{H} x_{T}(t)
$$

where $y(t)$ is the output of the beamformer, $x_{T}(t)$ is the output of the antenna elements, $w$ is the complex weight vector for the antenna element $=\left[w_{1}, w_{2}, \ldots, w_{L}\right]^{T}$ is $\in \boldsymbol{C}^{L \times I}$ beamforming complex vector. (. $)^{H}$ denotes the conjugate transpose (Hermitian transpose) of a vector or a matrix.

The weight vector at time $t+1$ for any system that uses the immediate gradient vector $\nabla J(t)$ for weight vector upgrading and evades the matrix inverse operation can express as follows:

$$
W(t+1)=W(t)+\frac{1}{2} \mu[\nabla J(t)]
$$

where $\mu$ is the step size parameter, which regulates the convergence speed and lies between 0 and 1 . While the smallest values of $\mu$ facilitate the high-quality estimation and sluggish concurrence, while the huge value may result in a rapid union. However, the constancy over the minimum value may disappear. Consider

$$
0<\mu<\frac{1}{\lambda}
$$

An instantaneous estimation of gradient vector is written as:

$$
\begin{gathered}
\nabla J(t)=-2 p(t)+2 R(t) W(t) \\
p(t)=d^{*}(t) x_{T}(t) \\
R_{y}=x_{T}(t) x_{T}{ }^{H}(t)
\end{gathered}
$$

An precise calculation of $\nabla J(t)$ is not possible because prior information on cross-correlation vector, $p$ and covariance matrix, $R_{y}$ of the measurement vector are required. By substituting (7) with (5), the weight vector is derived as follows:

$$
\begin{aligned}
W(t+1) & =W(t)+\mu[p(t)-R(t) W(t)] \\
= & W(t)+\mu x_{T}(t)\left[d^{*}(t)-x_{T}(t) W(t)\right] \\
= & W(t)+\mu x_{T} e^{*}(t)
\end{aligned}
$$

The desired signal can be further defined by the following three formulas:

$$
\begin{gathered}
y(t)=w^{H}(t) x_{T}(t) \\
e(t)=d(t) \cdot y(t) W(t+1) \\
=W(t)+\mu x_{T}(t) e^{*}(t)
\end{gathered}
$$

The covariance matrix, $R_{y}$ is constructed conventionally with unlimited snapshots. However, it is estimated by 
using limited snapshots signal in actual application. It can be expressed as:

$$
\begin{gathered}
R_{s}=\sigma_{s}^{2} a\left(\theta_{s}, \phi_{s}\right) a^{H}\left(\theta_{s}, \phi_{s}\right) \\
R_{i+n}=\sum_{i=1}^{I} \sigma_{i}^{2} a\left(\theta_{i}, \phi_{i}\right) a^{H}\left(\theta_{i}, \phi_{i}\right)+\sigma_{n}^{2} I d_{L} \\
R_{y}=R_{s}+R_{i+n}=\mathrm{E}\left[x_{T}(t) x_{T}^{H}(t)\right] \\
\sigma_{s}^{2}=E\left[\left|x_{s}(t)\right|^{2}\right] \\
\sigma_{i}^{2}=E\left[\left|x_{i}(t)\right|^{2}\right]
\end{gathered}
$$

where $R_{y}, \sigma_{s}^{2}, \sigma_{i}^{2}, \sigma_{n}^{2}, I d_{L}, R_{s}, R_{i+n}$ and $E[$.$] denotes, respectively, the L \times L$ theoretical covariance matri $\mathrm{x}$, power of the desired user signal, interference signal power, noise power, identity matrix, SOI covaria nce matrix, interference plus noise covariance matrix and expectation operator.

The common formulation of the MVDR beamformer that determines the $L \times 1$ optimum weight vector is the solution to the following constrained problem (Souden, Benesty \& Affes, 2010):

$$
\begin{aligned}
& W_{M V D R}=\arg _{W_{M V D R}} \min \left(W^{H} R_{y} W\right)=\min \mathrm{E}\left[|y(t)|^{2}\right] \\
& \Rightarrow \min _{w} P(\theta, \phi)=\left\{w^{H} R_{y} w\right\} \quad \text { s.t. } w^{H} a\left(\theta_{s}, \phi_{s}\right)=1
\end{aligned}
$$

where $P(\theta, \phi)$ denotes the mean output power, a beampattern can be given in $\mathrm{dB}$ as (Godara, 1997):

$$
\text { beampatter } n=20 \log _{10} \frac{|P(\theta, \phi)|}{\max |P(\theta, \phi)|}
$$

This technique minimizes the contribution of the interference signal by reducing the output noise and interference powers and ensuring the power of useful signal equals to "1" in the direction of useful signal $w^{H}$ $a\left(\theta_{s}, \phi_{s}\right)=1$. By using Lagrange multiplier, the MVDR weight vector that gives the solution for the equation (19) as per the following formula (Renzhou, 2007):

$$
w_{\mathrm{MVDR}}=\frac{R_{y}^{-1} a\left(\theta_{s}, \phi_{s}\right)}{a^{H}\left(\theta_{s}, \phi_{s}\right) R_{y}^{-1} a\left(\theta_{s}, \phi_{s}\right)}
$$

Inserting (20) into (11), the MVDR output is given as:

$$
\begin{aligned}
y(t) & =w^{H}(t) x_{T}(t) \\
& =w^{H} a\left(\theta_{s}, \phi_{s}\right) x_{s}(t)+w^{H} x_{i}(t) a\left(\theta_{i}, \phi_{i}\right)+w^{H} x_{n} \\
& =x_{s}(t)+w^{H} x_{i}(t) a\left(\theta_{i}, \phi_{i}\right)+w^{H} x_{n}
\end{aligned}
$$

The output signal power of the array as a function of the DOA estimation, using optimum weight vector from MVDR beamforming method (Haykin, 2013), it is given by MVDR spatial spectrum for angle of arrival (AoA) estimated by detecting the peaks in this angular spectrum as (Capon, 1969):

$$
P_{M V D R}(\theta)=\frac{1}{a^{H}\left(\theta_{s}, \phi_{s}\right) R_{y}^{-1} a\left(\theta_{s}, \phi_{s}\right)}
$$

Finally, the SINR is defined as: 


$$
\operatorname{SINR}=10 \log _{10} \frac{\sigma_{s}^{2}\left|w^{H} a\left(\theta_{s}\right)\right|^{2}}{\sum_{i=1}^{I} \sigma_{i}^{2}+\sigma_{n}^{2}}
$$

\section{Simulation Results and Analysis}

In this paper, $L$ antenna element in a CAAs configuration is arranged along some axis added to the beamformer system at the base station (BS). The CAA receives signals from various spatially separated users. The received signal at the CAA consists of a real user signal, co-channel interference, and background noise. To increase the output power of the desired signal and reduce the power of co-channel interference and noise, beamforming is employed at the BS. The ABF performance analysis shows an array of even and odd numbered elements separated by interelement spacing, $d$, at frequency $(F c)$ of $2.6 \mathrm{GHz}$, which is the spectrum band assigned for LTE services provider in Malaysia (Malaysian Communications and Multimedia Commission, 2011). To measure the performance of the MVDR algorithm for ABF applications with varying parameters like the number of antenna elements, the separation between adjacent elements, number of SNOIs, accuracy to distinguish interference in the location very close to the SOI, the number of snapshots $(n s)$, and noise power, $\sigma_{n}$. The goal is to analyze the effect of parameters mentioned above that achieve the best beamforming capabilities to form the main beam in the real user direction and place null in the direction of interference with highest SINR output. Four different scenarios are considered and the simulation parameters setting in this paper is shown in Table 1.

Table 1. Key simulation parameters of MVDR beamformer

\begin{tabular}{lc}
\hline Key system parameters & Values \\
\hline Array antenna configuration & Circular antenna array \\
Antenna type & Isotropic \\
Carrier frequency $(\mathrm{Fc})$ & $2.6 \mathrm{GHz}$ \\
Beam scanning range & $\pm 90^{\circ}$ (Azimuth) \\
Number of element $(L)$ & $5,8,11,16$ \\
Element spacing $(d)$ & $\lambda / 8, \lambda / 4, \lambda / 2, \lambda$ \\
SNOIs & $1,2,3,4$ \\
Noise power label $\sigma_{n}[\mathrm{~dB}]$ & $-50,-10,10,20$ \\
Snapshots $(n s)$ & $10,50,250,500$ \\
\hline
\end{tabular}

\subsection{The First Scenario}

The first simulation scenario depicted the results calculated by considering the distance between array elements set to be $d=\lambda / 2$ as usually used in the most MVDR algorithm, figure 2 illustrates MVDR angular spectrum plot for the estimated direction of all incoming signals implemented in this scenario. UCAA with $L=5,8,11$, and 16 elements, the additive noise is modeled as a complex zero-mean white Gaussian noise. Three interfering sources are assumed to have DOAs $-60^{\circ}, 0^{\circ}$ and $60^{\circ}$ respectively. The SOI is considered to be a plane wave from the pre-sumed direction $30^{\circ}$. The obtained results provide evidence that the received signals identified the SOI and SNOIs perfectly as assumed by producing peaks in the directions of $-60^{\circ}, 0^{\circ}, 30^{\circ}$ and $60^{\circ}$ azimuth angles and $90^{\circ}$ elevation angles respectively, which are computed using equation (22). Each one of these peaks represent the AoA of the incoming signal. When the number of $L$ increases, the peaks become sharper and improve the MVDR resolution for better detecting the incoming signal. Furthermore, the direction remains the same without any change. 


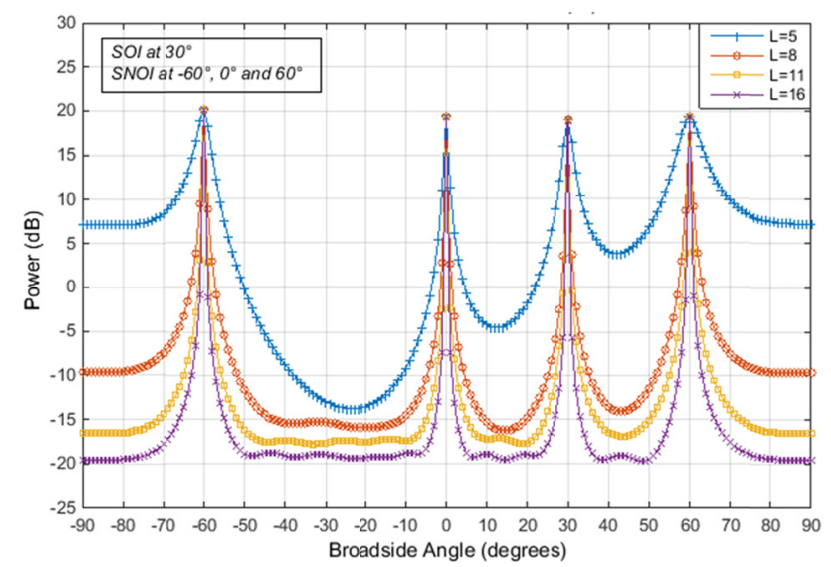

Figure 2. MVDR-DOA estimation analysis for varying number of elements.

With the direction of the incoming signals known or estimated, the next step is to use the MVDR BF technique to improve the signal performance of the desired target and nullifying interference directions. Figure 3 show a typical 2D linear and polar beampattern plots displayed in rectangular coordinate, which demonstrate the effect if the number of elements is increased for SOI at $\left(30^{\circ}, 90^{\circ}\right)$ and SNOIs at $\left(-60^{\circ}, 90^{\circ}\right),\left(0^{\circ}, 90^{\circ}\right),\left(60^{\circ}, 90^{\circ}\right)$ respectively. This simulation was repeated for 5, 8, 11, and 16 elements with an input SNR of $10 \mathrm{~dB}$ and 300ns. The plots observe that the MVDR successfully form nulls at each of the income interference sources, and it provides maximum gain to the look direction of the SOI. Moreover, increasing the number of elements results in the mainlobe beamwidth remain the same while the number of nulls in the pattern increases. The number of side lobes (SLs) increases whereas the level of the first and subsequent SLs decreases compared to the main beam. SLs represent power radiated in potentially unwanted directions so in a wireless communications system, SLs will contribute to the level of unwanted signal spread in the cell by a transmitter as well as the level of interference seen by a receiver when array of antennas are used. The mainlobe beamwidth (MLBw), maximum side lobe level (MSLL) that is closest to the main beam, maximum depth null (MDN) at interference direction and output SINR are shown in Table 2. On the other hand, the computing operations become more complex. Besides, as the number of $L$ increases, the cost of the design also increases due to the increasing of RF modules, A/D converters, and other components. This causes the operational power consumption to increase as well.
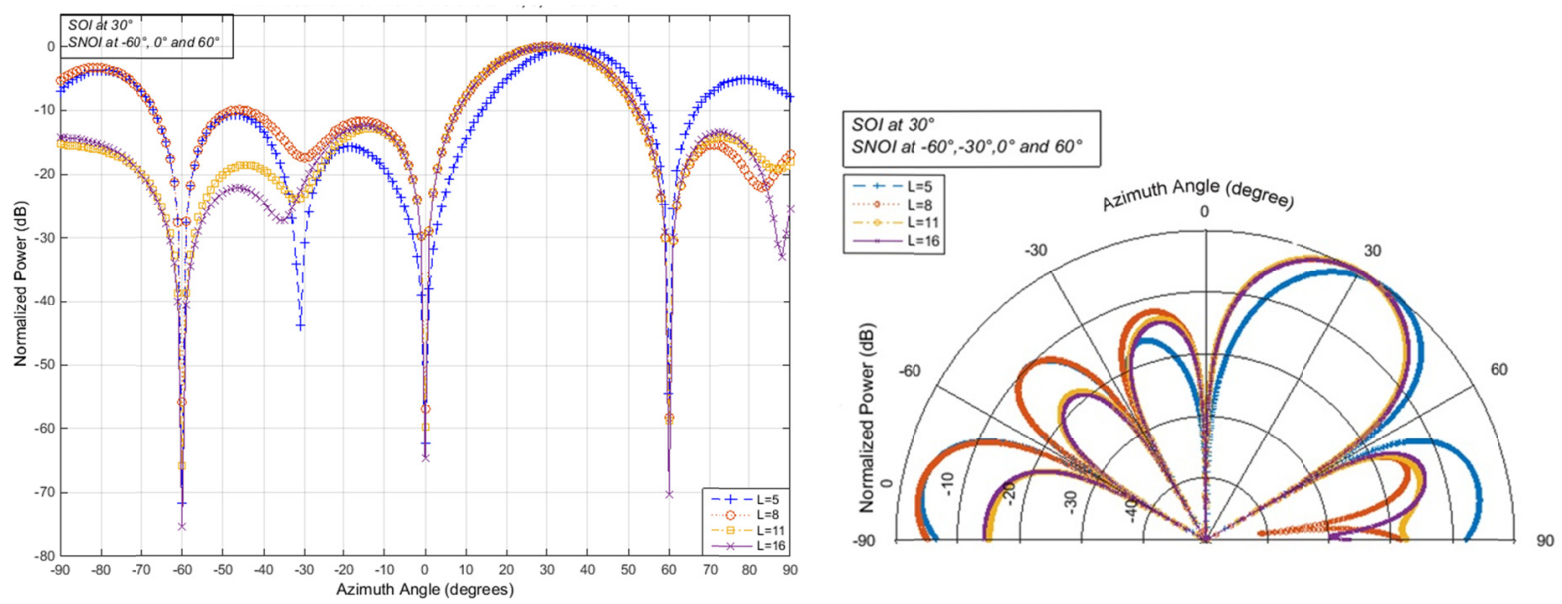

Figure 3. Beampattern analysis of MVDR varying $L=5,8,11$, and 16 with $d=\lambda / 2$. 
Table 2. MVDR performance analysis for SOI at $\left(30^{\circ}, 90^{\circ}\right)$ and SNOIs at $\left(-60^{\circ}, 90^{\circ}\right),\left(0^{\circ}, 90^{\circ}\right)$ and $\left(60^{\circ}, 90^{\circ}\right)$ with varying $L$

\begin{tabular}{lccccc}
\hline$L$ & $d[\mathrm{~m}]$ & MLBw $\left[^{\circ}\right]$ & MSLL [dB] & MDN [dB] & SINR [dB] \\
\hline 5 & $\lambda / 2[0.0577]$ & 60 & -5.0 & -71.0 & 52.1 \\
8 & $\lambda / 2[0.0577]$ & 60 & -11.6 & -58.3 & 52.9 \\
11 & $\lambda / 2[0.0577]$ & 60 & -12.8 & -65.7 & 55.7 \\
16 & $\lambda / 2[0.0577]$ & 60 & -12.4 & -75.3 & 63.3 \\
\hline
\end{tabular}

\subsection{The Second Scenario}

The distance between two elements in the design of an antenna array is an important factor. Second simulation scenario illustrates the results which calculated by considering $L=8$ elements with interelements spacing of $\lambda / 8$, $\lambda / 4, \lambda / 2$, and $\lambda$ for SOI at $\left(30^{\circ}, 90^{\circ}\right)$ and SNOIs at $\left(-60^{\circ}, 90^{\circ}\right),\left(0^{\circ}, 90^{\circ}\right),\left(60^{\circ}, 90^{\circ}\right)$.

Figure 4 demonstrate the effect of the element spacing on MVDR performance. It is found that for $\lambda / 8$ and $\lambda / 4$ the power of the mainlobe is less than optimum whereas the max power of the main beam is achieved by $d=\lambda / 2$ and $\lambda$, The coupling effects that appears when elements are spaced closely as shown in figure 4 for $d=\lambda / 8$ at $134^{\circ}$ with a power of $0 \mathrm{~dB}$. The side lobe level that is closest to the main beam for each interelement spacing has a height of $0,0,-0.9$, and -11.0 at $134^{\circ}, 118^{\circ}, 92^{\circ}$, and $-14^{\circ}$ respectively. Furthermore, if the spacing is less than $\lambda / 2$, it does not improve the MVDR performance in terms of resolution, and the coupling effects will be larger and tend to decrease as increase in the spacing. If the spacing is bigger than $\lambda / 2$, this causes grating lobes which is undesirable directions of maximum radiation that degrade the MVDR performance as well. Therefore, the elements spacing has to be far enough to avoid mutual coupling, and the spacing has to be $\leq \lambda / 2$ to prevent grating lobes. As the spacing between elements increases, the main beamwidth decreases resulting in higher directivity, the number of SLs also increases and the highest output SINR obtained by $\lambda / 2$ due to greatest interference suppression as depicted in Table 3 .
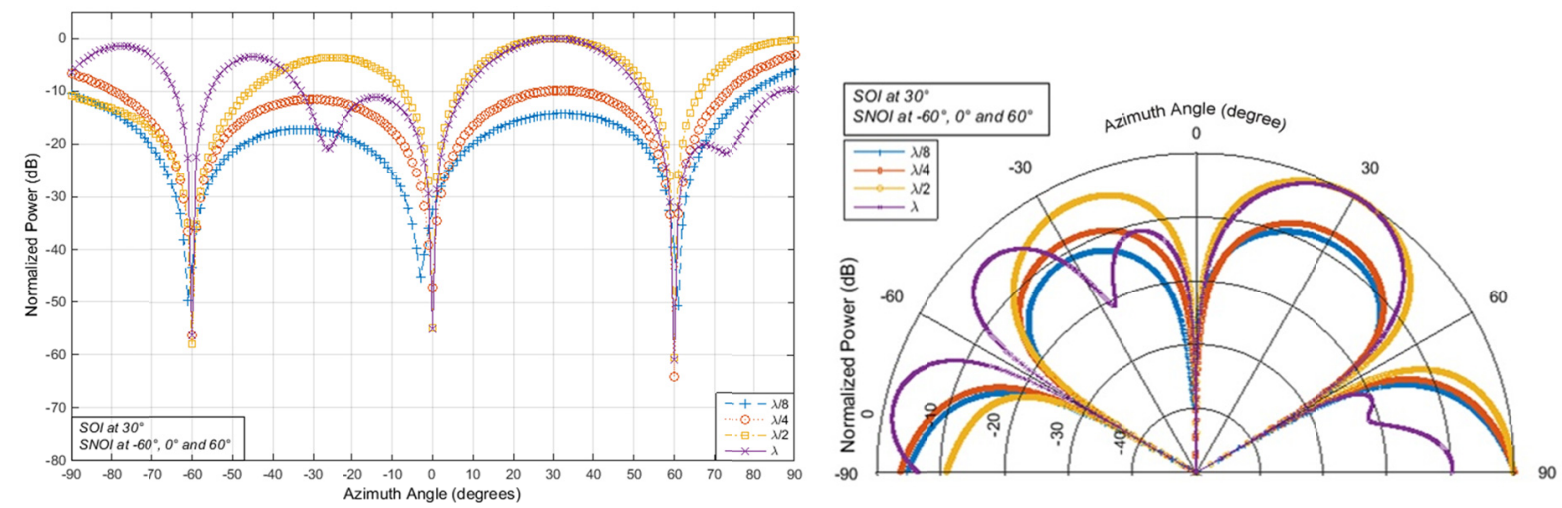

Figure 4. beampattern analysis of MVDR varying $d=\lambda / 8, \lambda / 4, \lambda / 2$ and $\lambda$ with $L=8$.

Table 3. MVDR performance analysis for SOI at $\left(30^{\circ}, 90^{\circ}\right)$ and SNOIs at $\left(-60^{\circ}, 90^{\circ}\right),\left(0^{\circ}, 90^{\circ}\right)$ and $\left(60^{\circ}, 90^{\circ}\right)$ with different $d$.

\begin{tabular}{cccccc}
\hline$L$ & $d[\mathrm{~m}]$ & MLBw $\left[{ }^{\circ}\right]$ & MSLL [dB] & MDN [dB] & SINR [dB] \\
\hline \multirow{4}{*}{8} & $\lambda / 8[0.0144]$ & 64 & 0 & -36.3 & 17.2 \\
& $\lambda / 4[0.0288]$ & 60 & 0 & -54.2 & 36.6 \\
\cline { 2 - 7 } & $\lambda / 2[0.0577]$ & 60 & -0.9 & -60.5 & 52.3 \\
\cline { 2 - 7 } & $\lambda[0.1153]$ & 60 & -11.0 & -60.9 & 50.1 \\
\hline
\end{tabular}

\subsection{The Third Scenario}

Using multiple antennas at the BS can reduce the effects of co-channel interference, multipath fading, and back-ground noise. Many BF algorithms have been devised to cancel interference sources that appear in the 
cellular system. MVDR algorithm has the ability to null the interferences without any distortion to the desired path.

The third simulation scenario demonstrates the MVDR behavior when the number of SNOIs increased. The subsequent MVDR pattern plotted with cancelation for all interferences is shown in figure 5. It shows the beampattern for an eight-element circular array in the presence of different AoAs for SOI and Interference signals (Int. Sigs.). In figure 5, the output of MVDR BF algorithm is illustrated against a different number of interference sources as tableted in Table 4. It can be seen that the performance of the MVDR is affected by the number of SNOIs as the interference source increases, the SINR decreases. In case of two interference sources, the deep null of $-68.7 \mathrm{~dB}$ compared to $-48.5 \mathrm{~dB}$ for 16 elements was found for a study conducted by (Saxena \& Kothari, 2014) based on conjugate gradient method ABF algorithm. For 4 interference sources, the MVDR was capable to form the mainlobe to reach a wanted user direction even for the closest interference to the user, which means better result is obtained when the angular separation between the SOI and SNOI increases.
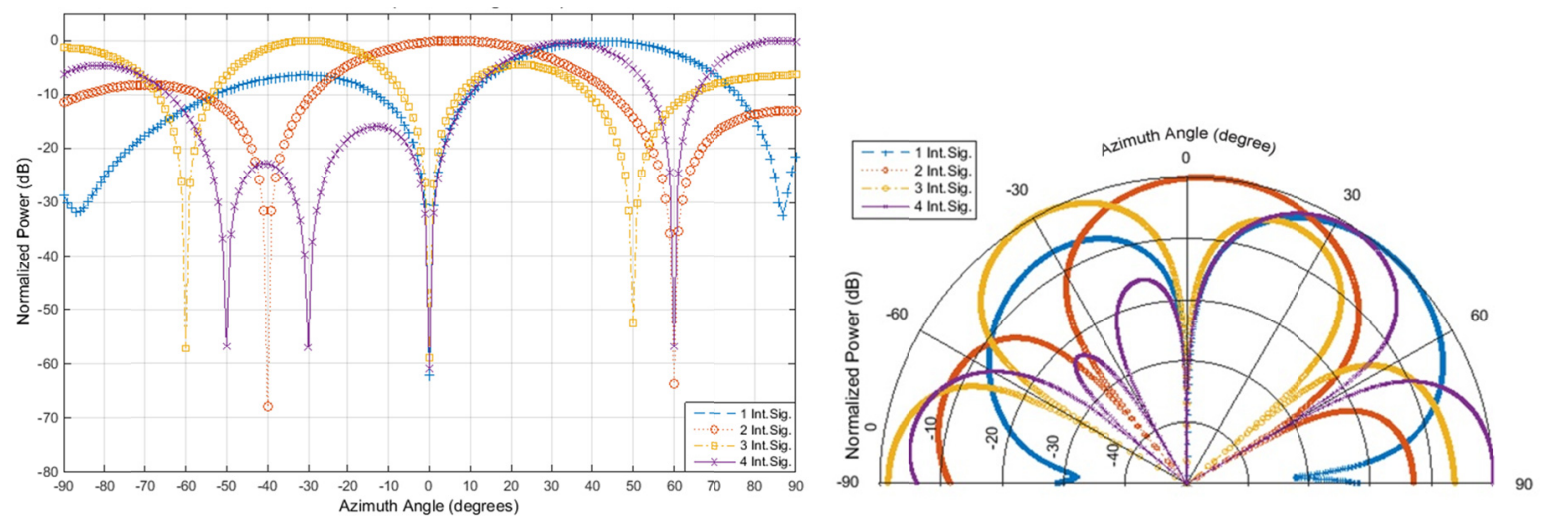

Figure 5. beampattern analysis of MVDR, $L=8$ and $d=\lambda / 2$ with different SOI and SNOIs AoAs.

Table 4. Comparison of SINR values for $L=8$ and $d=\lambda / 2$ with different SOI and SNOIs AoAs

\begin{tabular}{llllllll}
\hline$L$ & $d[\mathrm{~m}]$ & SOI $\left[^{\circ}\right]$ & SNOIs $\left[^{\circ}\right]$ & $\begin{array}{l}\text { MLBw } \\
{\left[{ }^{\circ}\right]}\end{array}$ & $\begin{array}{l}\text { MSLL } \\
{[\mathrm{dB}]}\end{array}$ & $\begin{array}{l}\text { MDN } \\
{[\mathrm{dB}]}\end{array}$ & $\begin{array}{l}\text { SINR } \\
{[\mathrm{dB}]}\end{array}$ \\
\hline \multirow{3}{*}{8} & \multirow{2}{*}{$\lambda / 2$} & 40 & 0 & 87 & -6.3 & -62.1 & 63.1 \\
& 0 & $-40,60$ & 100 & -8.1 & -67.7 & 62.1 \\
& {$[0.0577]$} & -20 & $-60,0,50$ & 60 & -1.3 & -57.6 & 49.1 \\
& 30 & $-50,-30,0,60$ & 60 & 0 & -60.2 & 46.8 \\
\hline
\end{tabular}

\subsection{The Fourth Scenario}

This scenario, compares the effect of various noise power levels, $\sigma_{n}$, and different number of snapshots, $n s$, on the performance of MVDR for a desired user at $\left(30^{\circ}, 90^{\circ}\right)$ and three interference signals at $\left(-60^{\circ}, 90^{\circ}\right),\left(0^{\circ}, 90^{\circ}\right)$ and $\left(60^{\circ}, 90^{\circ}\right)$ with $L=8, d=\lambda / 2$. Figure 6 gives the output beampattern with four different noise powers ranging from $-50 \mathrm{~dB}$ to $20 \mathrm{~dB}$. It can be seen that the four curves are noticeably very similar, the MVDR shows better SINR of $92.1 \mathrm{~dB}$ at the noise power level of $-50 \mathrm{~dB}$ with null all interference sources as demonstrated in Table 5 . The output SINR decreases as the noise power increases. However, the reduction (null power) at higher values of $\sigma_{n}$ is much less than at lower values of $\sigma_{n}$ and the MVDR performance deteriorates as the noise power increases.

Moreover, in figure 7, the beampattern of MVDR algorithm for analyzing the performance is illustrated against the number of ns and compared to the output SINR. It observed that the performance of the MVDR is effected by the number of snapshots where increasing number of ns resulting in more accurate resolution but also the computational time increases. In terms of required computational time, it is found that the required processing time for MVDR increases with the snapshot increase as displayed in Table 6. This simulation was operated on Intel ${ }^{\circledR}$ Core 2 Due CPU @ 3.0 GHz, 4 GB RAM run on windows 7-64 bit operating system. 

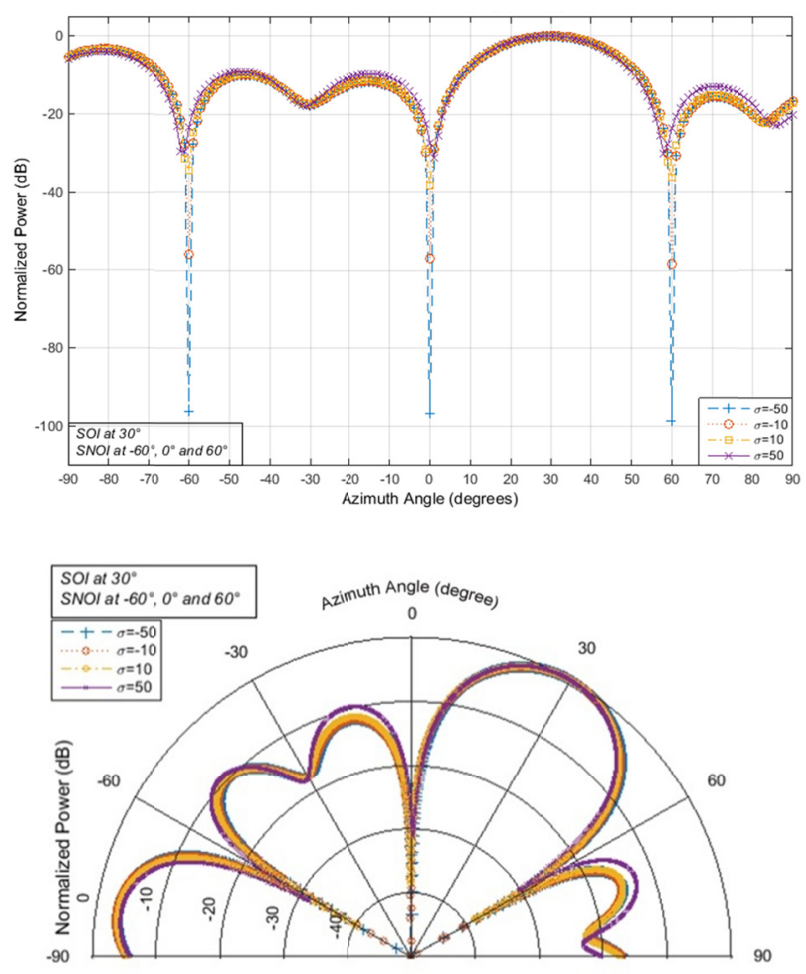

Figure 6. beampattern analysis of MVDR varying $\sigma_{n}=-50,-10,10$, and $20 \mathrm{~dB}$ with $L=8, d=\lambda / 2$
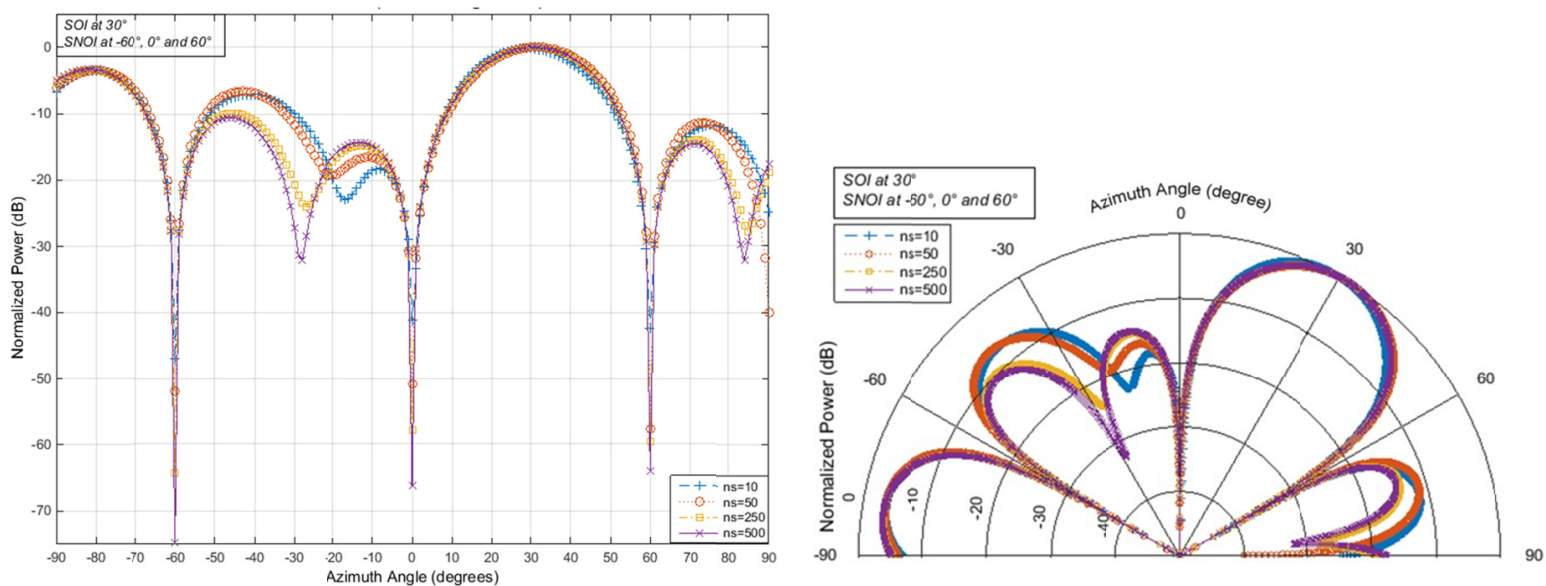

Figure 7. beampattern analysis of MVDR varying $n s=10,50,250$, and 500 with $L=8, d=\lambda / 2$

Table 5. MVDR performance analysis for SOI at $\left(30^{\circ}, 90^{\circ}\right)$ and SNOIs at $\left(-60^{\circ}, 90^{\circ}\right),\left(0^{\circ}, 90^{\circ}\right)$ and $\left(60^{\circ}, 90^{\circ}\right)$ with different $\sigma_{n}$

\begin{tabular}{ccccccc}
\hline$L$ & $d[\mathrm{~m}]$ & $\sigma_{n}[\mathrm{~dB}]$ & $\mathrm{MLBw}\left[{ }^{\circ}\right]$ & MSLL [dB] & MDN [dB] & SINR [dB] \\
\hline \multirow{4}{*}{8} & & -50 & 60 & -11.6 & -98.4 & 92.1 \\
& $\lambda / 2[0.0577]$ & -10 & 60 & -11.6 & -58.3 & 52.1 \\
& & 10 & 60 & -11.1 & -38.4 & 31.1 \\
& & 20 & 60 & -9.6 & -30.9 & 19.5 \\
\hline
\end{tabular}


Table 6. MVDR performance analysis for SOI at $\left(30^{\circ}, 90^{\circ}\right)$ and SNOIs at $\left(-60^{\circ}, 90^{\circ}\right),\left(0^{\circ}, 90^{\circ}\right)$ and $\left(60^{\circ}, 90^{\circ}\right)$ with different $n s$

\begin{tabular}{cccccccc}
\hline$L$ & $d[\mathrm{~m}]$ & $n s$ & MLBw $\left[^{\circ}\right]$ & MSLL [dB] & MDN [dB] & SINR [dB] & Time [Sec] \\
\hline \multirow{4}{*}{8} & & 10 & 60 & -11.7 & -47 & 38.0 & 1.98 \\
& $\lambda / 2$ & 50 & 60 & -11.2 & -57.6 & 47.7 & 2.10 \\
& {$[0.0577]$} & 250 & 60 & -13.9 & -64.2 & 55.0 & 2.27 \\
& & 500 & 60 & -14.4 & -74.7 & 61.8 & 2.7 \\
\hline
\end{tabular}

As seen in Table 5, the mainlobe beamwidth (MLBw) remain the same and the maximum side lobe level (MSLL) slightly changes as $\sigma_{n}$ increases, while the nulling level and SINR are strongly effected by $\sigma_{n}$ increases. In addition, Table 6 shows the SINR increases as ns increases owing to the increasing probability of finding a better solution. In other words, sharper and deeper nulls would be produced and hence improve the SINR by increasing number of snapshots. Finally, a summary of the impact of $L, d, \sigma_{n}$, and $n s$ on the MVDR performance for a trade-off analysis is presented in Table 7. In comparison, it is found that the MVDR basing LAA geometry has overall better performance for beampattern accuracy and highest SINR than CAA as reported in (Shahab, Zainun, Ali, Hojabri, \& Noordin, in press; Shahab, Zainun, Noordin, \& Balasim, 2016).

Table 7. MVDR trade-off analysis

\begin{tabular}{|c|c|c|c|}
\hline & Pros & Cons & Performance impact \\
\hline$L$ & $\begin{array}{l}\text { - More and deeper } \\
\text { nulls } \\
\text { - More degree of } \\
\text { freedom } \\
\text { - Higher SINR }\end{array}$ & $\begin{array}{l}\text { - More SLLs } \\
\text { - Larger size } \\
\text { - more costly } \\
\text { - Physical limitations on } \\
\text { Installation } \\
\text { - } \text { complexity }\end{array}$ & $\begin{array}{l}\text { Detter interference cancelation capabilities } \\
\text { Improved performance because of higher } \\
\text { SINR and narrower beams }\end{array}$ \\
\hline$d$ & $\begin{array}{ll}\text { - } & \text { Higher SINR } \\
\text { - } & \text { Cost-efficient }\end{array}$ & $\begin{array}{l}\text { - Grating lobes } \\
\text { - mutual coupling effects }\end{array}$ & $\begin{array}{l}\text { Grating lobes and mutual coupling have } \\
\text { negative impact on MVDR beamformer } \\
>\text { Wasted power in unnecessary direction }\end{array}$ \\
\hline$\sigma_{n}$ & $\begin{array}{ll}\text { - } & \text { Higher SINR } \\
\text { - deeper nulls }\end{array}$ & $\begin{array}{l}\text { - Lower SINR } \\
\text { - Reduce null level }\end{array}$ & $\begin{array}{l}>\text { Improved performance because of higher } \\
\text { SINR }\end{array}$ \\
\hline$n s$ & $\begin{array}{l}\text { - More accurate } \\
\text { resolution } \\
\text { - deeper nulls } \\
\text { - Higher SINR }\end{array}$ & - Time consuming & $\begin{array}{l}\text { Improved performance because of higher } \\
\text { SINR }\end{array}$ \\
\hline
\end{tabular}

\section{Conclusions}

This paper evaluates the MVDR algorithm for null steering of circular antenna arrays, to place deeply null at the interference sources in order to get maximum SINR for wanted direction. A number of computer simulations were performed with different numbers of antenna elements, different interelement spacings, different numbers of interference sources with varying angular separations between SOI and interferences, different levels of noise power, and different numbers of snapshots. The results obtained using MVDR algorithm has the best beam formed pattern in suppressing the interference and noise with best mainlobe shape when array elements are more. Meanwhile, the drawback is increasing cost, size and complexity. It is found that $0.5 \lambda$ is the best elements spacing for avoiding grating lobes, mutual coupling effects, and better null depth performance. MVDR rejects with a very low power level, and good accuracy can be obtained even in the case of multiple interferences. The noise level is an important factor that affects the MVDR performance. Increased number of snapshots results in higher SINR and more accurate main beampattern. The computation time can be further decreased if a higher signal processor is used. An ongoing research extends the results of this paper to enhance MVDR algorithm.

\section{Acknowledgments}

This research is sponsored by the research grant number (RDU 160351) funded by University Malaysia Pahang.

\section{References}

Balanis, C. A., \& Ioannides, P. I. (2007). Introduction to smart antennas. Arizona, USA: Morgan and Claypool 
Publishers.

Belloni, F., \& Koivunen, V. (2006). Beamspace transform for UCA: error analysis and bias reduction. IEEE Transactions on Signal Processing, 54(8), 3078-3089.

Capon, J. (1969). High-resolution frequency-wavenumber spectrum analysis. Proceedings of the IEEE, 57(8), 1408-1418.

Choi, R. L.-U., Murch, R. D., \& Letaief, K. (2003). MIMO CDMA antenna system for SINR enhancement. IEEE Transactions on Wireless Communications, 2(2), 240-249.

Cisco Visual Networking Index. (2014). Global mobile data traffic forecast update, 2013-2018 white paper. San Jose, CA, USA: Cisco Systems Inc.

Das, K. J., \& Sarma, K. K. (2012). Adaptive Beamforming for Efficient Interference Suppression Using Minimum Variance Distortionless Response. Paper presented at the International Conference on Advancement in Engineering Studies \& Technology.

Das, S. (2008). Smart antenna design for wireless communication using adaptive beam-forming approach. Paper presented at the IEEE Region 10 Conference, (TENCON'08). Hyderabad, India.

El, K. A., \& Champagne, B. (2008). Cooperative MIMO-beamforming for multiuser relay networks. Paper presented at the IEEE International Conference on Acoustics, Speech and Signal Processing, (ICASSP'08). Las Vegas, VA, USA.

El, Z. A. (2005). Smart antenna engineering. Norwood, MA, USA: Artech House, Inc.

Ericsson Mobility Report. (2015). On the Pulse of the Networked Society. Kista, Sweden,: Ericsson.

Godara, L. C. (1997). Application of antenna arrays to mobile communications. II. Beam-forming and direction-of-arrival considerations. Proceedings of the IEEE, 85(8), 1195-1245.

Godara, L. C. (2004). Smart antennas. Boca Raton: CRC press.

Gross, F. (2015). Smart antennas with matlab: principles and applications in wireless communication (2nd ed.): McGraw-Hill Professional.

Habets, E., Benesty, J., Cohen, I., Gannot, S., \& Dmochowski, J. (2010). New insights into the MVDR beamformer in room acoustics. IEEE Transactions on Audio, Speech, and Language Processing, 18(1), 158-170.

Halim, M. A. (2001). Adaptive array measurements in communications (1st ed.). Norwood, MA, USA: Artech House Publichers.

Han, R. (2009). Pattern Synthesis of Sparse Phased Array Antenna Using Genetic Algorithms. Modern Applied Science, 3(9), 91-94.

Haykin, S. (2013). Adaptive Filter Theory (4th ed.): Prentice Hall.

Hong, Y. W., Huang, W. J., Chiu, F. H., \& Kuo, C. C. J. (2007). Cooperative communications in resource-constrained wireless networks. IEEE Signal Processing Magazine, 24(3), 47-57.

Jacobsen, A. (2001). Smart antennas for dummies. Telenor R\&D Technical Report.

Khaldoon, A. O., Rahman, M. M., Ahmad, R. B., \& Hassnawi, L. A. (2014). Enhanced uniform linear array performance using modified minimum variance distortionless response beamformer algorithm. Paper presented at the 2nd International Conference on Electronic Design (ICED).

Khraisat, Y. S. (2012). Design of 4 elements rectangular microstrip patch antenna with high gain for $2.4 \mathrm{GHz}$ applications. Modern Applied Science, 6(1), 68-74.

Kum, D., Kang, D., \& Choi, S. (2014). Novel SINR-based user selection for an MU-MIMO system with limited feedback. ETRI journal, 36(1), 62-68.

Liberti, J. C., \& Rappaport, T. S. (1999). Smart antennas for wireless communications: IS-95 and third generation CDMA applications: Prentice Hall PTR.

Malaysian Communications and Multimedia Commission. (2011). SKMM-MCMC Annual Report. Retrieved from http://www.skmm.gov.my/skmmgovmy/media/General/pdf/

Manolakis, D. G., Ingle, V. K., \& Kogon, S. M. (2005). Statistical and adaptive signal processing: spectral estimation, signal modeling, adaptive filtering, and array processing. Norwood, MA, USA: Artech House, 
Inc.

Mathews, C. P., \& Zoltowski, M. D. (1994). Eigenstructure techniques for 2-D angle estimation with uniform circular arrays. IEEE Transactions on Signal Processing, 42(9), 2395-2407.

Pan, C., Chen, J., \& Benesty, J. (2014). Performance study of the MVDR beamformer as a function of the source incidence angle. IEEE/ACM Transactions on Audio, Speech, and Language Processing, 22(1), 67-79.

Rao, A. P., \& Sarma, N. (2014). Performance Analysis of Differential Evolution Algorithm based Beamforming for Smart Antenna Systems. International Journal of Wireless and Microwave Technologies (IJWMT), 4(1), $1-9$.

Renzhou, G. (2007). Suppressing radio frequency interferences with adaptive beamformer based on weight iterative algorithm. Paper presented at the Conference on Wireless, Mobile and Sensor Networks, (CCWMSN07), IET.

Saunders, R. S., \& Zavala, A. A. (2007). Antennas and propagation for wireless communication systems (2nd Ed.). Hershey, New York, USA: John Wiley \& Sons, Ltd.

Saxena, P., \& Kothari, A. (2014). Performance analysis of adaptive beamforming algorithms for smart antennas. IERI Procedia, Elsevier, 10, 131-137.

Serbetli, S., \& Yener, A. (2004). Transceiver optimization for multiuser MIMO systems. IEEE Transactions on Signal Processing, 52(1), 214-226.

Shahab, S. N., Zainun, A. R., Ali, H. A., Hojabri, M., \& Noordin, N. H. (in press). MVDR algorithm based linear antenna array performance assessment for adaptive beamforming application. Journal of Engineering Science \& Technology.

Shahab, S. N., Zainun, A. R., Noordin, N. H., \& Balasim, S. S. (2016). Assessment of MVDR Adaptive Beamforming Algorithm in Uniform Linear Arrays, Uniform Rectangular Arrays and Uniform Circular Arrays Configurations. ARPN Journal of Engineering and Applied Sciences, 11(6), 3911-3917.

Souden, M., Benesty, J., \& Affes, S. (2010). A study of the LCMV and MVDR noise reduction filters. IEEE Transactions on Signal Processing, 58(9), 4925-4935.

Stevanovic, I., Skrivervik, A., \& Mosig, J. R. (2003). Smart antenna systems for mobile communications. Lausanne, Switzerland: Laboratoire d'Electromagnetisme et d'Acoustique Ecole Polytechnique Federale de Lausanne.

Sun, C., Hirata, A., Ohira, T., \& Karmakar, N. C. (2004). Fast beamforming of electronically steerable parasitic array radiator antennas: theory and experiment. IEEE Transactions on Antennas and Propagation, 52(7), 1819-1832.

Van, T. H. L. (2002). Optimum array processing: part IV of detection, estimation, and modulation theory (1st Ed.). New York, USA: John Wiley \& Sons, Ltd.

Web ProForum Tutorials. (2006). Smart antenna systems. International Engineering Consortium. Retrieved from http://180.151.36.4/quality/TelecomBasics\%5Csmart_ant.pdf

Winters, J. H. (2000). WTEC Panel Report on Wireless Technologies and Information Networks, chapter 6. Smart Antennas International Technology Research Institute. Baltimore, MD, USA.

\section{Copyrights}

Copyright for this article is retained by the author(s), with first publication rights granted to the journal.

This is an open-access article distributed under the terms and conditions of the Creative Commons Attribution license (http://creativecommons.org/licenses/by/4.0/). 